\title{
Serological response to mRNA and inactivated COVID-19 vaccine in healthcare workers in Hong Kong: decline in antibodies 12 weeks after two doses
}

This article was published on 18 Oct 2021 at www.hkmj.org.
Hong Kong Med J 2021;27:380-3

https://doi.org/10.12809/hkmj219744
To the Editor-We previously reported serological vaccine arms were comparable except sex $(60.9 \%$ findings of 302 healthcare workers (HCWs) who and $38 \%$ female in CoronaVac and BNT162b2, completed two doses of mRNA (BNT162b2/ Comirnaty; Fosun-BioNTech Pharma) and inactivated COVID-19 vaccine (CoronaVac; Sinovac Life Sciences, Beijing, China). ${ }^{1}$ Both vaccines were found to be immunogenic in the majority of HCWs. The BNT162b2 resulted in a 11-fold higher level of anti-spike IgG (Abbott SARS-CoV-2 IgG II Quant assay, mean $=11572.6 \mathrm{AU} / \mathrm{mL}$ vs $1005.2 \mathrm{AU} / \mathrm{mL}$; $\mathrm{P}<0.001)$ and a higher surrogate neutralising antibody (sNAb) [GenScript cPass SARS-CoV-2 Surrogate Virus Neutralization Test Kit] positive rate $(100 \%$ vs $94.4 \% ; \mathrm{P}<0.001)$.

We report week 12 serological data of our cohort. Among 197 CoronaVac and 100 BNT162b2 recipients, baseline characteristics of the two respectively) [online supplementary Table 1]. There was no difference in anti-spike immunoglobulin G (IgG) positive rate at week 12 (98.5\% in CoronaVac vs $99 \%$ in BNT162b2; P=1) [Table 1]. Waning of IgG level was observed in both vaccine arms with a larger magnitude of decline in BNT162b2 (-72\% vs $-64.6 \%$; $\mathrm{P}<0.001)$. Despite the more pronounced decline, the median anti-spike IgG of BNT162b2 remained 11-fold higher than that of CoronaVac at week 12 (2840.25 AU/mL vs 253.60 AU/mL; P<0.001).

Decline in sNAb was also observed in both arms but the magnitude was significantly smaller in BNT162b2 $(-28.3 \%$ in CoronaVac vs $-2.3 \%$ in BNT162b2; $\mathrm{P}<0.001)$. Using the manufacturer's positive cut-off at $30 \%$ signal inhibition or above,

TABLE I. Antibody levels after vaccination with CoronaVac or BNTI62b2*

\begin{tabular}{|c|c|c|c|c|c|c|c|c|c|c|}
\hline & \multicolumn{3}{|c|}{ Corona Vac } & \multicolumn{3}{|c|}{ BNT162b2 } & \multicolumn{4}{|c|}{ P value $^{\dagger}$ (CoronaVac vs BNT162b2) } \\
\hline & $\begin{array}{c}\text { After } \\
\text { dose } 1(\geq 2 \\
\text { weeks) } \\
\text { "Sample } \\
1 \text { " }\end{array}$ & $\begin{array}{c}\text { After } \\
\text { dose } 2(\geq 2 \\
\text { weeks) } \\
\text { "Sample } \\
2 "\end{array}$ & $\begin{array}{c}\text { After dose } \\
2(\geq 12 \\
\text { weeks) } \\
\text { "Sample } \\
3 \text { " }\end{array}$ & $\begin{array}{c}\text { After } \\
\text { dose } 1(\geq 2 \\
\text { weeks) } \\
\text { "Sample } \\
1 \text { " }\end{array}$ & $\begin{array}{c}\text { After } \\
\text { dose } 2(\geq 2 \\
\text { weeks) } \\
\text { "Sample } \\
2 "\end{array}$ & $\begin{array}{c}\text { After dose } \\
2(\geq 12 \\
\text { weeks) } \\
\text { "Sample } \\
3 \text { " }\end{array}$ & $\underset{1}{\text { Sample }}$ & $\begin{array}{l}\text { Sample } \\
2\end{array}$ & $\underset{3}{\text { Sample }}$ & \\
\hline $\begin{array}{l}\text { No. of serum samples } \\
\text { available for analysis }\end{array}$ & & 197 & & & 100 & & & & & \\
\hline \multicolumn{11}{|l|}{$\begin{array}{l}\text { Abbott SARS-CoV-2 } \\
\text { lgG }\end{array}$} \\
\hline $\begin{array}{l}\text { Antibody positive } \\
\text { results }\end{array}$ & $\begin{array}{c}128 \\
(65.0 \%)\end{array}$ & $\begin{array}{c}196 \\
(99.5 \%)\end{array}$ & $\begin{array}{c}194 \\
(98.5 \%)\end{array}$ & $98(98.0 \%)$ & $\begin{array}{c}100 \\
(100 \%)\end{array}$ & 99 (99.0\%) & $<0.001$ & 1 & 1 & $\begin{array}{l}\text { Fisher's } \\
\text { exact test }{ }^{\S}\end{array}$ \\
\hline $\begin{array}{l}\text { Antibody level, } \\
\text { AU/mL, median } \\
(\mathrm{IQR})^{\ddagger}\end{array}$ & $\begin{array}{l}84.40 \\
(36.50- \\
161.80)\end{array}$ & $\begin{array}{c}721.30 \\
(449.20- \\
1142.80)\end{array}$ & $\begin{array}{c}253.60 \\
(163.70- \\
403.00)\end{array}$ & $\begin{array}{l}1156.45 \\
(542.88- \\
1816.58)\end{array}$ & $\begin{array}{l}9704.15 \\
(6187.30- \\
14616.48)\end{array}$ & $\begin{array}{c}2840.25 \\
(1849.93- \\
4522.68)\end{array}$ & $<0.0001$ & $<0.0001$ & $<0.0001$ & $\begin{array}{l}\text { Wilcoxon } \\
\text { rank-sum } \\
\text { test" }\end{array}$ \\
\hline \multicolumn{11}{|l|}{$\begin{array}{l}\text { GenScript cPass } \\
\text { surrogate neutralising } \\
\text { antibody }\end{array}$} \\
\hline $\begin{array}{l}\text { Antibody positive } \\
\text { results }\end{array}$ & $32(16.2 \%)$ & $\begin{array}{c}186 \\
(94.4 \%)\end{array}$ & $\begin{array}{c}123 \\
(62.4 \%)\end{array}$ & $93(93.0 \%)$ & $\begin{array}{c}100 \\
(100 \%)\end{array}$ & 99 (99.0\%) & $<0.0001$ & 0.0182 & $<0.0001$ & $\begin{array}{l}\text { Fisher's } \\
\text { exact test }\end{array}$ \\
\hline $\begin{array}{l}\text { Antibody level, \% } \\
\text { signal inhibition, } \\
\text { median (IQR) }\end{array}$ & $\begin{array}{l}12.61 \\
(2.43- \\
25.18)\end{array}$ & $\begin{array}{l}69.30 \\
(52.07- \\
52.07)\end{array}$ & $\begin{array}{l}35.27 \\
(23.60- \\
51.41)\end{array}$ & $\begin{array}{l}68.29 \\
(51.59- \\
82.56)\end{array}$ & $\begin{array}{l}97.34 \\
(95.95- \\
98.87)\end{array}$ & $\begin{array}{l}95.58 \\
(88.71- \\
97.94)\end{array}$ & $<0.0001$ & $<0.0001$ & $<0.0001$ & $\begin{array}{l}\text { Wilcoxon } \\
\text { rank-sum } \\
\text { test }\end{array}$ \\
\hline
\end{tabular}

Abbreviations: IgG = immunoglobulin G; IQR = interquartile range; SARS-CoV-2 = severe acute respiratory syndrome coronavirus 2

Data are shown in No. (\%), unless otherwise specified

A P value of $<0.05$ was considered statistically significant

Mean was used in the previous letter

Fisher's exact test's null hypothesis: there is no dependence between positive/negative result and vaccine types

Wilcoxon rank-sum test's null hypothesis: there is no difference in central tendency (median) of the readings between vaccine type 
significantly more CoronaVac recipients had lost their sNAb at week 12 (94.4\% sNAb positive at week $2,62.4 \%$ at week 12) whereas $99 \%$ of BNT162b2 recipients remained $\mathrm{sNAb}$ positive. Throughout the three time points, BNT162b2 arm had higher levels of anti-spike IgG and sNAb $(\mathrm{P}<0.001)$ [Fig].

Among the $286 \mathrm{HCWs}$ who had positive sNAb after two doses of vaccine, 64 had lost their sNAb while 222 had sustained sNAb at week 12 . Sustained $\mathrm{sNAb}$ at week 12 were associated with younger age, BNT162b2 and higher antibody at any time point (Table 2). Multivariate logistic regression analysis showed that only higher IgG and sNAb level at 2 weeks after the second dose were significantly associated with sustained SNAb at week 12 (online supplementary Table 2).

These results demonstrate rapid antibody decline after both mRNA and inactivated vaccine with a more durable sNAb in the BNT162b2 arm. However, further studies are needed to clarify the impact of waning antibody on vaccine efficacy and protection against severe infection.

\section{Author contributions}

Concept or design: All authors.
Acquisition of data: All authors

Analysis or interpretation of data: All authors.

Drafting of the manuscript: JST Zee.

Critical revision of the manuscript for important intellectual content: All authors.

All authors had full access to the data, contributed to the study, approved the final version for publication, and take responsibility for its accuracy and integrity.

\section{Conflicts of interest}

All authors have disclosed no conflicts of interest.

\section{Acknowledgement}

The authors acknowledge the excellent work and contributions by staff at the Clinical Pathology Laboratory of Hong Kong Sanatorium \& Hospital.

\section{Funding/support}

This letter received no specific grant from any funding agency in the public, commercial, or not-for-profit sectors.

\section{Ethics approval}

This study obtained ethics approval (Ref RC-2021-07) from the Research Ethics Committee of the Hong Kong Sanatorium \& Hospital Medical Group. (a)

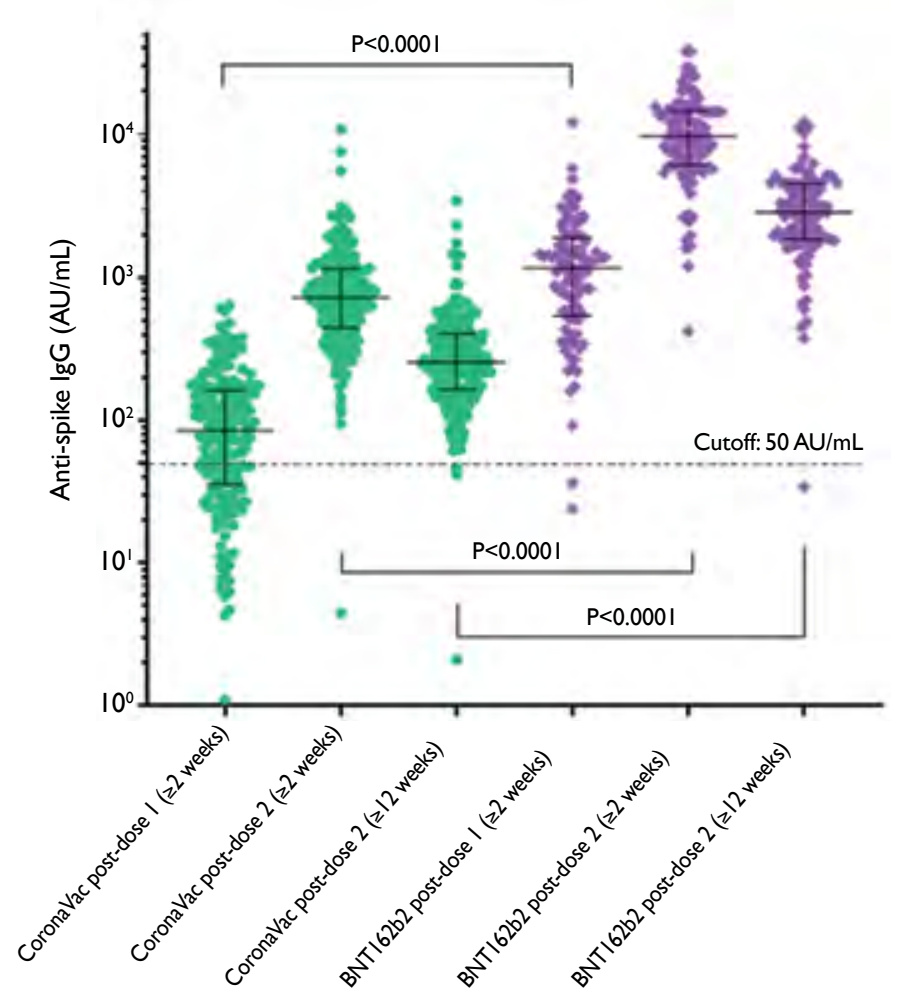

(b)

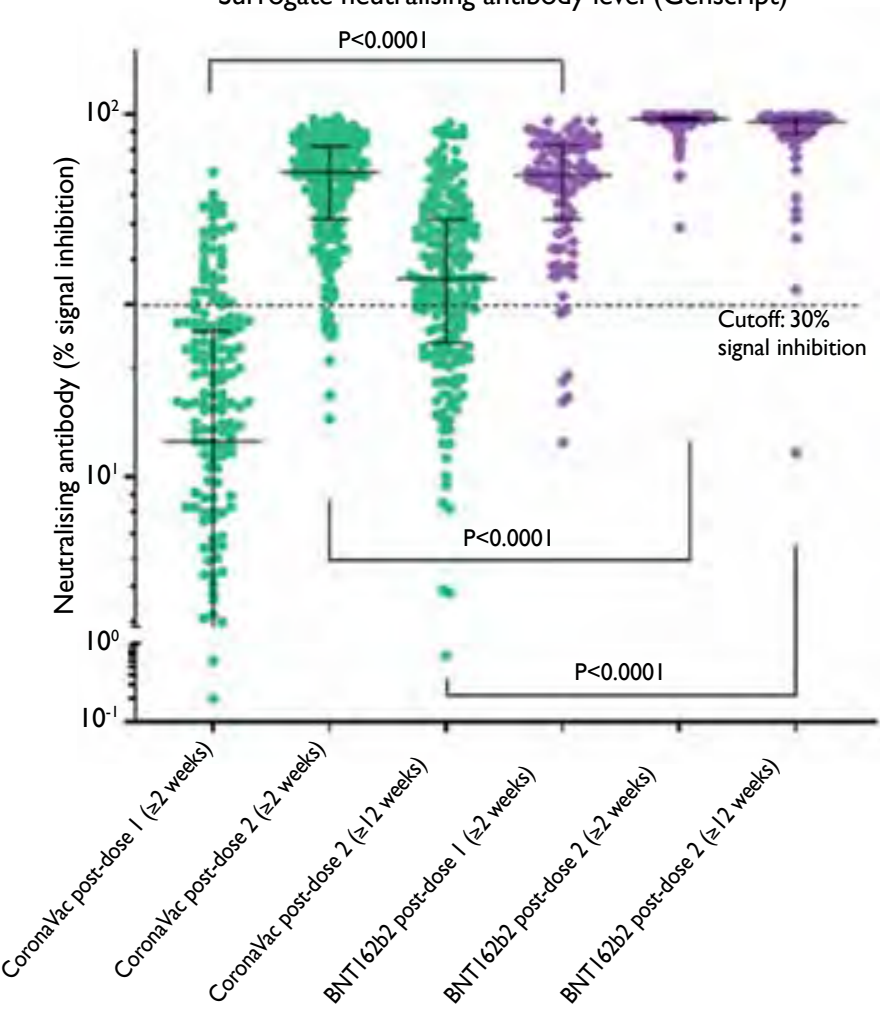

FIG. (a) Anti-spike immunoglobulin G (IgG) level; (b) surrogate neutralising antibody level after dose I and 2 of CoronaVac and BNTI62b2. Each dot represents the antibody level of a participant after dose I or dose 2 of CoronaVac or BNTI62b2 
TABLE 2. Factors associated with sustained or lost surrogate neutralising antibody (sNAb) at week $12^{*}$

\begin{tabular}{|c|c|c|c|}
\hline & $\begin{array}{l}\text { sNAb lost at week } 12 \\
(n=64)\end{array}$ & $\begin{array}{l}\text { sNAb sustained at } \\
\text { week } 12(n=222)\end{array}$ & P value $^{\dagger}$ \\
\hline Sex & & & 0.0749 \\
\hline Male & $23(35.9 \%)$ & $110(49.5 \%)$ & \\
\hline Female & $41(64.1 \%)$ & $112(50.5 \%)$ & \\
\hline Age, y & $50.75 \pm 10.12$ & $47.21 \pm 10.20$ & 0.0154 \\
\hline Vaccine type & & & $<0.0001$ \\
\hline CoronaVac & $63(98.4 \%)$ & $123(55.4 \%)$ & \\
\hline BNT162B2 & $1(1.6 \%)$ & 99 (44.6\%) & \\
\hline \multicolumn{4}{|l|}{ Medical co-morbidites } \\
\hline No. of medical co-morbidities & & & 0.9386 \\
\hline None & $49(76.6 \%)$ & $158(71.2 \%)$ & \\
\hline 1 & $9(14.1 \%)$ & 40 (18\%) & \\
\hline 2 & $4(6.3 \%)$ & $15(6.8 \%)$ & \\
\hline 3 & $2(3.1 \%)$ & $8(3.6 \%)$ & \\
\hline 4 & 0 & 0 & \\
\hline 5 & 0 & $1(0.5 \%)$ & \\
\hline Diabetes mellitus & $4(6.3 \%)$ & $10(4.5 \%)$ & 0.5233 \\
\hline Hypertension & $6(9.4 \%)$ & $23(10.4 \%)$ & 1 \\
\hline Hyperlipidaemia & $6(9.4 \%)$ & $26(11.7 \%)$ & 0.8219 \\
\hline Cardiovascular disease & $1(1.6 \%)$ & $1(0.5 \%)$ & 0.3981 \\
\hline Stroke & 0 & 0 & - \\
\hline Asthma & 0 & $4(1.8 \%)$ & 0.5782 \\
\hline Chronic renal disease & 0 & $1(0.5 \%)$ & 1 \\
\hline Chronic liver disease or cirrhosis & $3(4.7 \%)$ & $4(1.8 \%)$ & 0.1890 \\
\hline History of malignancy & $2(3.1 \%)$ & $8(3.6 \%)$ & 1 \\
\hline Systemic lupus erythematosus & 0 & 0 & - \\
\hline Other autoimmune disease & $1(1.6 \%)$ & $6(2.7 \%)$ & 1 \\
\hline \multicolumn{4}{|l|}{ Medication } \\
\hline Steroid & 0 & $1(0.5 \%)$ & 1 \\
\hline Non-steroid immunosuppressant & 0 & $1(0.5 \%)$ & 1 \\
\hline \multicolumn{4}{|l|}{ Abbott SARS-CoV-2 IgG (AU/mL) } \\
\hline After dose 1 ( $\geq 2$ weeks) & $70.50 \pm 77.48$ & $232.45 \pm 982.83$ & $<0.0001$ \\
\hline After dose 2 ( $\geq 2$ weeks) & $454.95 \pm 363.50$ & $1952.25 \pm 8033.10$ & $<0.0001$ \\
\hline After dose 2 ( $\geq 12$ weeks) & $158.45 \pm 94.83$ & $654.20 \pm 2347.80$ & $<0.0001$ \\
\hline \multicolumn{4}{|c|}{$\begin{array}{l}\text { GenScript cPass surrogate neutralising antibody } \\
\text { (\% signal inhibition) }\end{array}$} \\
\hline After dose 1 ( $\geq 2$ weeks) & $7.81 \pm 18.64$ & $35.28 \pm 52.14$ & $<0.0001$ \\
\hline After dose 2 ( $\geq 2$ weeks) & $55.00 \pm 23.44$ & $88.53 \pm 22.45$ & $<0.0001$ \\
\hline After dose 2 ( $\geq 12$ weeks) & $21.57 \pm 9.90$ & $70.25 \pm 48.17$ & $<0.0001$ \\
\hline \multicolumn{4}{|l|}{ (CoronaVac only) } \\
\hline \multicolumn{4}{|l|}{ Abbott SARS-CoV-2 IgG (AU/mL) } \\
\hline After dose 1 ( $\geq 2$ weeks) & $72.10 \pm 78.45$ & $115.50 \pm 129.55$ & 0.0036 \\
\hline After dose 2 ( $\geq 2$ weeks) & $458.20 \pm 364.30$ & $986.20 \pm 711.90$ & $<0.0001$ \\
\hline After dose 2 ( $\geq 12$ weeks) & $159.50 \pm 90.80$ & $356.20 \pm 252.60$ & $<0.0001$ \\
\hline \multicolumn{4}{|c|}{$\begin{array}{l}\text { GenScript cPass surrogate neutralising antibody } \\
\text { (\% signal inhibition) }\end{array}$} \\
\hline After dose 1 ( $\geq 2$ weeks) & $7.56 \pm 18.73$ & $16.16 \pm 20.60$ & 0.0052 \\
\hline After dose 2 ( $\geq 2$ weeks) & $55.64 \pm 24.17$ & $78.02 \pm 18.10$ & $<0.0001$ \\
\hline After dose 2 ( $\geq 12$ weeks) & $21.70 \pm 9.86$ & $47.55 \pm 24.22$ & $<0.0001$ \\
\hline
\end{tabular}

Abbreviations: IgG = immunoglobulin G; SARS-CoV-2 = severe acute respiratory syndrome coronavirus 2; sNAb = surrogate neutralising antibody

* Data are shown as mean \pm standard deviation or No. (\%), unless otherwise specified

† No. of positive test results was tested using Fisher's exact test or Chi squared test. Means were tested using $t$ test. A P value of $<0.05$ was considered statistically significant 
1,2 Jonpaul ST Zee *, FRCPath, FHKAM (Medicine)

${ }^{1}$ Kristi TW Lai, MMedsc (HKU)

${ }^{1}$ Matthew KS Ho, MMedSc (HKU)

${ }^{1}$ Alex CP Leung, MMedSc (HKU)

${ }^{3}$ LH Fung, MPhil

${ }^{3}$ WP Luk, MPhil

${ }^{4}$ LF Kwok, BSc (Nursing)

${ }^{4} \mathrm{KM}$ Kee, MPH

${ }^{2}$ Queenie WL Chan, BScN, FHKAN (Medicine-Infection Control)

1,2 SF Tang, FHKCPath, FHKAM (Pathology)

${ }^{1}$ Edmond SK Ma, MD, FRCPath

${ }^{5}$ KH Lee, MMedSc (HKU), FHKAM (Community Medicine)

${ }^{5}$ CC Lau, MB, BS, FHKAM (Emergency Medicine)

1,2,5 Raymond WH Yung, MB, BS, FHKCPath

\footnotetext{
${ }^{1}$ Department of Pathology, Hong Kong Sanatorium \& Hospital, Hong Kong

2 Infection Control Team, Hong Kong Sanatorium \& Hospital, Hong Kong

${ }^{3}$ Medical Physics and Research Department, Hong Kong Sanatorium \&

Hospital, Hong Kong

${ }^{4}$ Quality and Safety Division, Hong Kong Sanatorium \& Hospital, Hong

Kong

${ }^{5}$ Hospital Administration, Hong Kong Sanatorium \& Hospital, Hong Kong

*Corresponding author: jonpaul.st.zee@hksh.com
}

\section{Reference}

1. Zee JS, Lai KT, Ho MK, et al. Serological response to mRNA and inactivated COVID-19 vaccine in healthcare workers in Hong Kong: preliminary results. Hong Kong Med J 2021;27:312-3. 